\section{Situar a hiperatividade e déficit de atenção em Portugal: dimensões sociais, históricas e éticas de um tema emergente na saúde global}

\author{
Situating attention deficit and hyperactivity in \\ Portugal: social, historical, and ethical dimensions \\ of an emerging global health issue
}

\begin{abstract}
Situar la hiperactividad y déficit de atención en Portugal: dimensiones sociales, históricas y éticas de un tema emergente en la salud global
\end{abstract}

Angela Marques Filipe 1

doi: 10.1590/0102-311X00056420

\section{Resumo}

A perturbação de hiperatividade e déficit de atenção (PHDA) é considerada um dos problemas de comportamento e neurodesenvolvimento mais frequentes nas crianças e adolescentes em idade escolar, tanto em Portugal como a nível mundial. Além disso, a categorização diagnóstica da PHDA e a prescrição de psicoestimulantes como tratamento de primeira linha têm sido não só objeto de pesquisa científica e validação clínica, mas também alvo de polêmica e crítica social, sobretudo à luz do conceito de medicalização. Apesar do seu aspecto midiático e relevância em domínios tão diversos quanto os da educação, do medicamento, da saúde mental e dos apoios psicossociais, existe uma lacuna profunda na caracterização das dimensões históricas, socioéticas e institucionais da PHDA em Portugal. Aliando dados historiográficos e etnográficos à análise documental e da mídia, este artigo procura responder a esse desafio, traçando a trajetória social da PHDA neste país - desde a emergência da "hiperatividade" nos anos 1970/1980 ao debate público e politico sobre a prescrição de psicoestimulantes. Dessa perspectiva interdisciplinar e por meio do estudo do caso português, procura contextualizar-se a definição, validação e ampliação da PHDA como parte de um processo dinâmico e socialmente situado na interseção de sistemas diagnósticos e farmacêuticos globais, contingências institucionais e socioeconômicas e, ainda, politicas públicas e especificidades locais. Discute-se, por fim, a forma como o caso da PHDA em Portugal contribui para o desenvolvimento de novas linhas de reflexão e de pesquisa interdisciplinar que permitem repensar o escopo social da saúde mental e da saúde global.

Transtorno do Déficit de Atenção com Hiperatividade; Política Pública; Saúde Global; Saúde Mental; Ciências Sociais
Correspondência

A. M. Filipe

Department of Sociology, McGill University. 3460 McTavish, Montréal/QC - H3A 1Y2, Canada. angela.filipe@mcgill.ca

${ }^{1}$ McGill University, Montréal, Canada. 


\section{Introdução}

A perturbação de hiperatividade e déficit de atenção (PHDA) é considerada como um dos transtornos do comportamento e neurodesenvolvimento mais frequentemente diagnosticada nas crianças e jovens em idade escolar, com uma prevalência mundial estimada de $5 \%$ a $7 \%$ em estudos recentes 1,2. No entanto, cientistas sociais alertaram para o fato de o estudo socioepidemiológico da PHDA levantar diversas questões empíricas e metodológicas, tanto no plano nacional como numa perspectiva global 3,4. Essa advertência tem sido sublinhada também pelos especialistas dessa área em Portugal, onde não existem, até esta data, dados concretos e confiáveis sobre a prevalência e distribuição da PHDA, argumentando que esse é um diagnóstico complexo e clinicamente variável 5,6. Essa ambiguidade e complexidade formam um dos aspectos visíveis de um processo de definição, validação e, por vezes, contestação da categoria médica da PHDA e da utilização de psicoestimulantes no seu tratamento, processo este que atravessa a esfera privada do encontro clínico e das necessidades individuais e familiares, e a esfera coletiva das políticas públicas de saúde, de regulação dos psicofármacos e dos apoios educativos e psicossociais. Dessa forma, a PHDA é reconhecida como um diagnóstico cada vez mais frequente na população em idade escolar e que vem suscitando apreensão e atenção midiática, mas cujas dimensões históricas, socioéticas e institucionais não têm sido sistematizadas e documentadas na literatura ou estão ainda por explorar 3,4,7.

Atendendo a essa lacuna no conhecimento, este artigo procura traçar a evolução histórica e o panorama contemporâneo da PHDA em Portugal, com o objetivo de contextualizar os aspetos sociais do seu diagnóstico, tratamento, epidemiologia e enquadramento institucional. Inspirando-se na literatura da antropologia e sociologia médicas, da ética empírica e da história da psiquiatria, este artigo propõe uma perspectiva conceitual sobre a PHDA vista não apenas como uma categoria médica fixa e universalizante, mas como parte de um processo de definição, validação e negociação que é dinâmico e socialmente situado 3,7. Seguindo a proposta de Arjun Appadurai 8 de olhar para a "vida social das coisas", este texto procura mapear a trajetória social da PHDA no caso português, desde a sua emergência como "objeto" de avaliação e intervenção médicas até a sua (re)configuração como tema de debate público e político. Nessa perspectiva, analisa-se a forma como a vida social da PHDA interseta dinâmicas globais no campo da saúde, medicina e da classificação diagnóstica, as particularidades da sua história e epidemiologia sociais em Portugal, assim como as práticas clínicas, a defesa de direitos e as contingências socioeconômicas e institucionais que afetam a disponibilidade e diversidade dos modelos de cuidado existentes. Discutem-se, por fim, os atuais desafios e implicações futuras da PHDA no contexto português e a forma como este caso nos permite, ao mesmo tempo, situar e repensar as dimensões sociais da saúde pública e mental no contexto global de uma perspectiva coletiva e interdisciplinar.

\section{Enquadramento do projeto e desenho de pesquisa}

Este artigo tem como base um estudo qualitativo que foi conduzido entre 2010 e 2015 na Escola de Economia e Ciências Política de Londres [London School of Economics and Political Science] (LSE) e no Colégio Real de Londres [King's College London] (Reino Unido), com um financiamento de pesquisa de doutoramento atribuído pela Fundação para a Ciência e Tecnologia (FCT-Portugal). Esse estudo procurou investigar: (1) o processo pelo qual a PHDA se tornou num objeto de problematização e intervenção médicas e (2) a forma como os profissionais de saúde, nos seus encontros com famílias e jovens, abordam o diagnóstico, tratamento e implicações sociais da PHDA. Com o objetivo de responder a essas questões e para preencher uma lacuna tanto na disponibilização como na integração de dados nesse domínio em Portugal, o estudo aqui descrito incluiu três componentes de pesquisa.

Primeiro, uma revisão crítica da literatura científica sobre a PHDA nos contextos nacional e internacional. Essa revisão conduziu a uma busca específica de artigos sobre os seus aspetos sociais, éticos, neurocientíficos e institucionais, incluindo o papel desempenhado por diversos atores (i.e., médicos, familiares, indústria) no desenvolvimento de abordagens, práticas profissionais e representações sociais do seu diagnóstico e tratamento. Essa revisão permitiu ainda o desenvolvimento de literacia 
conceitual sobre dimensões clínicas e técnicas da PHDA, assim como o planejamento da pesquisa historiográfica e etnográfica subsequente.

Segundo, uma análise historiográfica sobre a saúde mental e o desenvolvimento infantil em Portugal com base em pesquisa de arquivo. Essa análise incluiu uma revisão da literatura e o desenvolvimento de uma bibliografia anotada com base em três volumes sobre a história da psicologia, da psiquiatria e da saúde mental infantil em Portugal 9,10,11. Após esse mapeamento inicial, foi realizada uma pesquisa de arquivo no Centro de Documentação Bissaya Barreto (2011) e na Biblioteca Nacional (2013), com o objetivo de recolher dados sobre a saúde mental infantil em Portugal no século XX, incluindo artigos científicos, notas biográficas e anais de congressos médicos publicados entre as décadas de 1920 e 1990. Acrescentou-se ainda a análise documental de relatórios clínicos, epidemiológicos e ministeriais dos organismos relevantes publicados desde então.

Terceiro, um estudo etnográfico de uma consulta de hiperatividade ligada ao Hospital Universitário de Coimbra. O trabalho de campo, realizado em 2012, envolveu: (a) a observação (não participante) de 46 consultas de especialidade (incluindo avaliações psicológicas), (b) a análise textual e visual dos questionários de avaliação utilizados nestas consultas e (c) entrevistas com cinco profissionais de saúde que estiveram diretamente envolvidos na avaliação e gestão da PHDA, além de observações de dois encontros científicos onde estiveram presentes. O estudo recebeu aprovação do Comitê de Ética da LSE e da equipa clínica local que verificou as folhas de informação e consentimento. Todos os materiais (incluindo transcrições de entrevistas e notas de observação) foram codificados, anonimizados e arquivados confidencialmente, procedendo-se à remoção ou alteração de detalhes e características pessoais que pudessem permitir a identificação de participantes e/ou terceiros. Acrescentou-se, por fim, uma comunicação pessoal com a fundadora do principal grupo de apoio a famílias e crianças com PHDA e a revisão seletiva de artigos, relatórios e notícias publicados desde 2015, no sentido de ampliar e atualizar a base de dados existente.

\section{A emergência do diagnóstico de "hiperatividade": da categorização psiquiátrica à descrição clínica}

A história da saúde mental infantil em Portugal caracteriza-se pelo surgimento de diversas escolas de pensamento e especialidades médicas (incluindo a médico-pedagogia, psiquiatria, psicanálise, psicologia e pediatria do desenvolvimento) que implicam, por sua vez, diferentes abordagens clínicas e terapêuticas aos problemas de comportamento e desenvolvimento nas crianças e jovens ${ }^{12}$. Num país marcado por mais de quatro décadas de ditadura, importa salientar que os médicos desempenharam um papel social, político e filantrópico muito ativo nesse domínio ao longo do século XX. Esse papel refletiu-se ainda no processo de criação do Serviço Nacional de Saúde Português (SNS) durante o período de transição democrática do final da década de 1970 13,14. A par desse panorama sociopolítico, a implementação tardia de políticas públicas e de enquadramento legal e institucional no domínio da saúde mental tornou-se ainda mais visível na área da saúde mental infantil e juvenil 15,16. Artigos científicos nesse domínio retratam, ainda, um panorama de resistência à implementação de sistemas de classificação e categorização diagnóstica na prática clínica até os anos 1980, altura em que a $5 \underline{a}$ versão da Classificação Internacional das Doenças (CID) da Organização Mundial da Saúde (OMS) foi traduzida e implantada no país 17,18 .

Em função dessas circunstâncias históricas é surpreendente constatar que a disciplina de neuropsiquiatria infantil foi oficialmente reconhecida em Portugal no auge do regime salazarista, em 1959, na mesma época em que o foi nos Estados Unidos 12. Da mesma forma, as primeiras referências a critérios comportamentais semelhantes ao diagnóstico da PHDA apareceram na literatura clínica portuguesa do final dos anos 1970 e $1980^{3}$. Num compêndio publicado em 1977 pelo então designado Centro de Saúde Mental e Infantil de Lisboa e pelo Instituto Nacional de Assistência Psiquiátrica, a disfunção cerebral mínima aparece descrita como sendo uma síndrome que incluía impulsividade, hipercinésia, suscetibilidade emocional, dificuldade de concentração, imaturidade comportamental e baixa tolerância à frustração ${ }^{19}$. No panorama internacional, assistiu-se à publicação da terceira edição do Manual de Diagnóstico e Estatística das Perturbações Mentais em 1980 (ou DSM-III, na versão original inglesa), que veio a revolucionar as bases teóricas da psiquiatria e a uniformizar os seus critérios 
de classificação diagnóstica no nível internacional, como descreve o antropólogo Andrew Lakoff 20. Especificamente, o DSM-III redefiniu a desatenção, a impulsividade e a hiperatividade como a tríade sintomática na qual assenta o diagnóstico por déficit de atenção e hiperatividade e os seus três subtipos: predominantemente desatento, predominantemente impulsivo/hiperativo e de tipo combinado. Foi nessa época que a designação de hiperatividade começou a ser destacada no campo da medicina e da psicologia em Portugal. Num artigo de 1986, Rebelo 21 descreveu o quadro clínico da hiperatividade como um conjunto de reações indiscriminadas e impulsivas a diferentes estímulos, no qual as crianças se mostravam irrequietas e incapazes de cumprir tarefas em diferentes contextos (p.ex.: na escola e em casa) e com um impacto negativo no seu rendimento escolar e no funcionamento social.

Desde então, a noção de "hiperatividade" tem-se destacado e popularizado como sinônimo funcional da PHDA em Portugal, onde as consultas de especialidade foram designadas por "consultas de hiperatividade" 22,23. O fato de a hiperatividade ter alcançado maior destaque do que a componente de déficit de atenção está também relacionado com a adoção das categorias e critérios do DSM publicado pela Associação de Psiquiatria Americana (APA) 24, e a sua eventual tradução e adaptação para diferentes populações clínicas e os seus respectivos contextos culturais e linguísticos ${ }^{3}$. Esse processo de adoção e adaptação da categoria diagnóstica implicou alguma variação semântica e conceitual, mesmo dentro do idioma português: a terminologia adotada no Brasil é do transtorno do déficit de atenção $\mathrm{com} / \mathrm{sem}$ hiperatividade (TDAH), em Portugal foi adotada a designação de perturbação de hiperatividade e déficit de atenção (PHDA) 7. A tradução do diagnóstico de PHDA inverteu a ordem dos seus critérios e elementos clínicos, o que pode resultar numa sobrevalorização dos comportamentos externalizantes de hiperatividade em detrimento dos impactos globais e socialmente menos visíveis da desatenção 25,26 . Além disso, a identificação e sinalização de comportamentos hiperativos relacionados com a PHDA têm sido marcadas por uma diferenciação de gênero associada a representações sociais de rapazes irrequietos ou impertinentes que externalizam os seus impulsos, ao contrário das moças, como foi extensamente abordado por Ilina Singh 27,28. O surgimento da hiperatividade e a PHDA em Portugal reflete, desse modo, um processo de adoção local de sistemas diagnósticos e de adaptação de categorias médicas que é moldado por contingências históricas, dinâmicas sociopolíticas e atribuições profissionais específicas, tal como é sugerido na literatura dos estudos sociais da ciência 29,30 .

\section{A validação do diagnóstico e tratamento da PHDA com psicoestimulantes: entre contestação e ativismo}

Após o surgimento da noção de hiperatividade nos anos 1980 em Portugal, assistiu-se nas décadas seguintes à sua validação na comunidade médica local e à introdução, entre a década de 1990 e o início de 2000, de medicamentos psicoestimulantes no tratamento da PHDA 3,7. Durante esse período, a PHDA foi sendo definida entre a comunidade médica local como um transtorno legítimo do comportamento e como uma disfunção do neurodesenvolvimento, clinicamente relevante e para a qual deve ser disponibilizado tratamento psicofarmacológico. Os marcos históricos desse processo incluem a organização de um importante simpósio de neurologia pediátrica, em 1996 31, e uma edição especial da revista Psychologica dedicada à hiperatividade e aos transtornos de atenção, em 1998 32, na sequência da proposta de Russell Barkley 33 para que fosse criada uma teoria unificadora da PHDA. No plano internacional, publicavam-se também os resultados do primeiro estudo multimodal do tratamento da PHDA financiado pelo Instituto Nacional de Saúde Mental dos Estados Unidos (National Institute of Mental Health - NIHM), o que levou ao estabelecimento de um consenso diagnóstico e terapêutico por meio do qual se estabeleceu uma maior eficácia e preferência pelo uso de psicoestimulantes no tratamento da PHDA 34. Apesar de os psicoestimulantes à base de metilfenidato terem sido comercializados e subsidiados pelo SNS Português no início da década de 2000 35, vale a pena salientar que estes foram introduzidos como parte do tratamento da PHDA na década anterior, sujeitos à receita médica especial, tal como indica o Decreto-Lei no 15/1993 36. Relatos médicos da época indicam que as farmácias de hospitais centrais dispunham de uma quantidade limitada de embalagens, graças a um mecanismo de importação direta feita da Suíça, e que quando o medicamento estava esgotado algumas 
famílias portuguesas se dirigiam com as suas receitas à Espanha, país vizinho e também membro da então Comunidade Econômica Europeia 37.

Esse processo de validação e criação de um consenso em torno do diagnóstico e tratamento da PHDA foi acompanhado por alguma contestação inicial e pelo aparecimento de um grupo de apoio e defesa de direitos. Tal como em outros países, debatia-se naquela época a neurociência e a especificidade etiológica da PHDA, a validade clínica e transcultural das categorias e critérios diagnósticos definidos pela APA por meio do DSM e, ainda, as implicações éticas e sociais da prescrição de psicoestimulantes no contexto pediátrico 38,39,40. Em 1999, por exemplo, um pediatra português publicou um comentário que advertia para os limites da categorização psiquiátrica de comportamentos problemáticos na criança como distúrbio, assim como para os riscos da sobreutilização de medicamentos psicoestimulantes no seu tratamento 41. Foi também no final dos anos 1990 que a mãe de duas crianças diagnosticadas com PHDA resolveu criar um grupo de apoio e defesa de direitos, a Associação Portuguesa da Criança Hiperativa (APdCH - ver detalhes em http://www.apdch.net). Durante esse período de transição, a ausência de conscientização e reconhecimento público da PHDA, bem como a falta de informação e de acesso a cuidados, colocaram as famílias numa posição relativamente ambígua e difícil. Nesse sentido, a APdCH foi criada com o objetivo de responder às necessidades de pais e famílias e de sensibilizar a opinião pública portuguesa: "alguns pais não tinham acesso a medicação ou não conseguiam comportar os custos [e] havia professores que não compreendiam [esta questão] e maltratavam as nossas crianças” 3 (p. 123).

Por um lado, foi nessa época que a PHDA começou a ser validada por membros da comunidade médica e contestada por alguns profissionais de saúde e educação. Por outro, foi durante esse período que a PHDA foi gradualmente considerada uma condição legítima e merecedora de atenção médica, apoio familiar e intervenção terapêutica. Não surpreende, portanto, que relatos de médicos e especialistas dessa época mobilizassem evidências científicas e clínicas para argumentar contra a posição daqueles que atribuíam a desatenção e hiperatividade à PHDA a falta de vontade e mau comportamento da criança, responsabilizando as famílias e as práticas educativas dos pais 42 . Como sugerido pelo historiador Charles Rosenberg ${ }^{43}$, receber um diagnóstico médico e psiquiátrico pode resultar numa situação que é socialmente paradoxal. Se o diagnóstico pode ajudar a legitimar problemas de comportamento e necessidades familiares facilitando o acesso a cuidados especializados e apoios sociais, ele pode também ser visto com uma forma de categorização médica que resulta, por vezes, na discriminação e estigmatização sociais de quem vive com esse diagnóstico. Indo além do interesse sociológico nessa problemática como caso exemplar da medicalização do comportamento e da sociedade, tal como descreveram Peter Conrad 44 e outros cientistas sociais 45 , sugere-se aqui que essa dinâmica de legitimação e contestação (ou de ativismo e resistência) assinala um dos aspetos mais peculiares e também persistentes da vida social da PHDA.

\section{Modelos de cuidado e intervenção: complementaridade e contingência}

De acordo com a literatura médica, os tratamentos farmacológicos e as intervenções psicoeducativas devem ser combinados na gestão da PHDA. Com o objetivo de responder às necessidades específicas de cada criança e da sua família, esta abordagem multimodal poderá incluir estratégias comportamentais, de intervenção com pais e famílias, bem como adaptações em sala de aula e outras formas de terapia 46,47,48. Pode dizer-se, assim, que o leque de repostas e abordagens terapêuticas à PHDA em Portugal se situa entre dois modelos de cuidado e intervenção: o modelo médico e farmacológico e o modelo psicossocial e pedagógico. Embora esses modelos devam, em teoria, complementar-se como parte de uma abordagem multimodal e integrada, na prática eles podem deparar-se com alguns desafios e contingências institucionais. Por exemplo, na clínica onde foi realizado este trabalho, o diagnóstico de PHDA era feito por uma equipe multidisciplinar de pediatras e psicólogos em colaboração com psicólogos clínicos e do desenvolvimento, pedopsiquiatras, terapeutas da fala e assistentes sociais e de educação. Essa equipe mantinha uma relação de proximidade com os pais, famílias e professores (os quais estão diretamente envolvidos no preenchimento de questionários sobre o comportamento da criança em casa e no meio escolar) além dos psicólogos escolares e/ou privados, a fim de recolher informações pertinentes sobre as crianças e jovens que eram referenciados para avaliação. No entan- 
to, essas relações não serão sempre lineares, tal como se verificou em duas observações da consulta de PHDA neste estudo.

Se num primeiro caso a professora se queixou do comportamento de um aluno, um menino com dez anos de idade, e pediu à mãe que prosseguisse com o tratamento prescrito, num segundo caso, uma mãe constatou junto à psicóloga que a professora da sua filha de oito anos se recusava a dar-lhe a medicação. Essas observações ilustram posições normativas distintas relativamente ao reconhecimento e tratamento da PHDA, retratando dois casos extremos: no primeiro, uma relação colaborativa e de aceitação e, no segundo, uma relação polarizada em que está patente uma posição de resistência ao tratamento. Esse retrato tem sido descrito por sociólogos e historiadores norte-americanos como sendo parte de um processo de medicalização da educação e da infância 49,50. Todavia, esse processo tende a ser mais ambivalente e menos polarizado no terreno, variando não só ao longo do tempo (por exemplo, na medida em que aumentam os níveis de sensibilização e informação sobre a PHDA em meio escolar) mas também de acordo com o contexto cultural, geográfico e sociohistórico em que se insere. Nesse sentido, e com base num estudo aprofundado sobre a utilização de psicoestimulantes no contexto escolar chileno, Rojas Navarro \& Vrecko 51 defenderam que esses fenômenos devem ser encarados como sendo parte de um "enredamento" farmacêutico que assume formas locais específicas e que assenta, poderemos aqui acrescentar, em relações sociais e culturas institucionais que são localmente situadas.

O panorama é igualmente complexo e, por vezes, fragmentado no que toca às medidas de apoio educativo e às abordagens pedagógicas neste domínio. Por exemplo, a revisão do Decreto-Lei no 3/2008 52 do Ministério da Educação que redefiniu os termos da educação especial em Portugal, recomenda uma combinação de estratégias e meios de intervenção que incluem o apoio pedagógico personalizado, protocolos de avaliação adaptados e um currículo individual com conteúdos de aprendizagem adequados. O requerimento necessário para aceder a esses apoios e adaptações deve ser feito pelos pais e corroborado por um relatório médico que terá de ser reconhecido pelo/a professor/a ou diretor/a escolar em causa. Esse modelo de educação especial e o seu enquadramento legal comportam algumas limitações burocráticas e institucionais no que diz respeito a relatórios e formulários, os quais podem nem sempre ser acessíveis aos pais causando atrasos também na atribuição de outros apoios, tais como o subsídio por frequência de estabelecimento de educação especial 53 . Mais ainda, esse modelo assenta no pressuposto da diferenciação em meio escolar e pode não estar à altura da complexidade suscitada por problemas de comportamento e dificuldades de aprendizagem associadas. A esse respeito, Pais et al. 54 defenderam que a diferenciação e medicalização de distúrbios comportamentais como PHDA poderão ocultar falhas estruturais dos atuais modelos pedagógicos e das práticas escolares em Portugal.

Por sua vez, o modelo de intervenção médico e farmacológico na PHDA tem enfrentado outro tipo de desafios no contexto português. O metilfenidato foi aprovado pela Autoridade Nacional do Medicamento e Produtos de Saúde (Infarmed) e subsidiado pelo Estado Português em 2003 35. Durante a década seguinte (2003-2013), estiveram disponíveis apenas três tipos de metilfenidato com as marcas registadas Rubifen, Ritalina LA e Concerta as quais contêm, respetivamente, uma fórmula mais barata de efeito de curto prazo e uma fórmula mais dispendiosa, com efeito prolongado 55,56. Apesar desses medicamentos estarem subsidiados, o país atravessou nessa mesma década um grave período de recessão econômica após um pedido de resgate financeiro em 2011 57. Essa situação justificou a implementação de medidas de austeridade pela troika que conduziram, por sua vez, à subida da taxa de desemprego e ao agravamento das desigualdades sociais no acesso aos cuidados de saúde, como descrito no relatório do Observatório Português dos Sistemas de Saúde ${ }^{58}$. Foi precisamente durante esse período que se denunciou uma ruptura de estoque do Rubifen, que o Infarmed atribuiu a problemas na cadeia de distribuição do medicamento e que um pediatra associou, por sua vez, ao custo consideravelmente mais baixo deste medicamento quando comparado ao Concerta 59. Ao mesmo tempo, o Jornal $i$ divulgou dados da consultora internacional de marketing farmacêutico IMS-Health que indicavam um aumento de quase $80 \%$ nas vendas de metilfenidato em Portugal entre 2007 e 2011 60. A divulgação simultânea desses dados internacionais e daquela ruptura de estoque pela comunicação social portuguesa originou uma primeira vaga de atenção midiática sobre a comercialização e prescrição de psicoestimulantes no país. 
O ano de 2012 marcou, assim, uma virada importante na trajetória social do diagnóstico da PHDA e do seu tratamento com psicoestimulantes como temas de debate público e de crítica profissional. Essa virada veio a culminar na publicação de um manifesto em defesa de uma "abordagem não medicalizante nem patologizante da educação" por um grupo de profissionais na área da educação e saúde mental 61. Os signatários defendiam que uma abordagem predominantemente neurobiológica e farmacológica à PHDA pode obscurecer as suas determinantes ambientais, educacionais e sociais, contribuindo não só para uma responsabilização indevida dos indivíduos e das famílias, mas também para uma tendência ao sobrediagnóstico e à sobreprescrição de psicoestimulantes nesse contexto 3 . Essas vozes juntaram-se às de pediatras portugueses que já tinham expressado a sua preocupação relativa a lacunas estruturais na prestação de apoios psicopedagógicos e de terapias de longo prazo, as quais contribuem para uma predominância das intervenções farmacológicas no tratamento da PHDA 62. Outros alertaram, ainda, para os riscos de uma intervenção médica inadequada fundamentada num modelo patológico dos transtornos do desenvolvimento e comportamento na população em idade escolar (ao invés de um modelo dimensional), o que pode motivar um excesso de zelo diagnóstico e terapêutico 63 .

Quanto aos dados oficiais existentes, o primeiro Relatório do Gabinete de Informação e Planeamento Estratégico do Infarmed sobre a evolução das taxas de prescrição de metilfenidato na faixa etária dos 5 aos 19 anos foi publicado em 2015, e revelou mais do que uma duplicação destas taxas entre 2010 e 2014 atingindo as 13,4 doses diárias definidas (DDD) por mil habitantes em Portugal continental 35. Essa tendência de crescimento replicou-se entre os anos de 2013 e 2016, ano em que as vendas de metilfenidato atingiram as 377.331 embalagens em Portugal, segundo dados disponibilizados no último relatório do Programa Nacional para a Saúde Mental da Direção-Geral da Saúde (DGS) 64. Convém salientar, todavia, que durante esse período foram comercializados três genéricos desse medicamento em Portugal, com base num procedimento descentralizado de autorização de introdução no mercado, previsto na diretiva europeia 2001/83/EC 65, seguindo-se a autorização da atomoxetina em 201435 e da lisdexanfetamina em 2019 66. Porém, o relatório de 2019 do Conselho Nacional de Saúde (CNS) sobre a saúde mental em Portugal aponta para uma tendência inversa de diminuição do consumo de metilfenidato no quinquênio precedente ${ }^{67}$. Durante esse período, a divulgação de dados e relatórios técnicos, pela imprensa portuguesa, sobre a venda de psicoestimulantes contribuiu para a galvanização e politização do debate sobre as repostas à PHDA, gerando artigos noticiosos e de opinião, além de debates parlamentares sobre as implicações sociais da prescrição de psicoestimulantes em contexto escolar e pré-escolar 6,68,69,70.

\section{Enquadramento e ampliação da PHDA: da epidemiologia às novas abordagens clínicas e terapêuticas}

Em Portugal, os relatórios oficiais sobre saúde mental têm evidenciado dificuldades na obtenção de dados sistemáticos e confiáveis nesta área, especialmente no domínio da epidemiologia psiquiátrica e da saúde mental da criança e do adolescente 16,71. O primeiro relatório nacional sobre a epidemiologia da saúde mental em Portugal, publicado em 2013 e patrocinado pelo World Mental Health Surveys Initiative da OMS, referia uma taxa de prevalência da PHDA de 0,4\% e 1,5\% ao longo da vida 72. Todavia, este estudo deparou-se com inúmeras dificuldades na recolha de dados e inquiriu apenas a população adulta, na qual o diagnóstico tem sido feito com menor frequência e para a qual a prescrição de psicoestimulantes foi autorizada apenas a partir de 2011 35. Nessas circunstâncias, os profissionais de saúde basearam-se tanto em estudos regionais e em dados de países culturalmente aproximados como em estudos internacionais e estimativas globais resultantes de metanálises da literatura 1,2 . Por exemplo, num volume publicado em 2014 são indicadas taxas de prevalência rondando 4\% e 5\%, com base em estudos locais realizados nos anos 1990 e no início da década de 2000 48, ao passo que um segundo livro, do mesmo ano, afirma que havia 80 mil crianças em idade escolar com PHDA em Portugal, baseando-se numa taxa internacional estimada entre 5\% e $8 \%$ 22. Torna-se, assim, difícil aferir em que medida esses dados fornecem uma estimativa confiável e atualizada da sua prevalência na população portuguesa e, por conseguinte, a distribuição relativa das taxas de prescrição e consumo de psicoestimulantes na subpopulação em idade escolar. 
Além disso e ao contrário de países como o Reino Unido e os Estados Unidos, onde o Instituto Nacional de Excelência em Saúde e Cuidados (National Institute for Health and Care Excellence NICE) e a Associação Americana de Pediatria (AAP) publicaram normas de orientação no diagnóstico e tratamento da PHDA 73,74, não existe uma norma de orientação clínica integrada em Portugal até esta data. Existem apenas recomendações gerais para a saúde mental infanto-juvenil nos cuidados de saúde primários e do ponto de vista da referenciação e da identificação precoce 71,75. Essas recomendações fornecem um sistema de referência para médicos de família, embora a prerrogativa da avaliação, diagnóstico e intervenção na PHDA recaia sobre as especialidades médicas da pedopsiquiatria e da (neuro)pediatria nos cuidados secundários. Por todas essas razões, o relatório do Programa Nacional para a Saúde Mental (PNSM) de 2017 previu a criação de um grupo de trabalho sobre a prescrição de psicofármacos em idade pediátrica para caracterizar a realidade atual e definir recomendações neste campo ${ }^{64}$. Foi ainda reiterado, no final de 2018, que a Ordem dos Médicos e a DGS publicariam, num futuro próximo, um guia de normas de orientação clínica que sintetizará os conhecimentos e as boas práticas a seguir na avaliação e tratamento da PHDA 76, no seguimento de um parecer da Ordem sobre o tratamento da PHDA emitido pelo Colégio de Psiquiatria da Infância e Adolescência no ano anterior 77 .

Para além do SNS, importa salientar que vários centros e unidades de saúde no setor privado oferecem consultas de especialidade dedicadas à PHDA, representando 39\% das prescrições de metilfenidato no país em 2015, segundo o relatório do Infarmed antes mencionado 35. Deve-se notar, todavia, que essas unidades e os seus profissionais assumiram um papel importante no desenvolvimento e na aplicação de protocolos clínicos de avaliação e de terapias cognitivo-comportamentais, preenchendo algumas das lacunas encontradas no setor público. Esse papel envolve, por exemplo, o desenvolvimento e aperfeiçoamento de novas estratégias de intervenção, tais como o neurofeedback e a terapia cognitiva-comportamental, a formação de pais ou treino parental, assim como a revisão de práticas clínicas no diagnóstico e tratamento da PHDA em adultos 22,48,78. Do ponto de vista ético e sociológico, coloca-se assim a hipótese de que a PHDA possa ser avaliada e tratada mais frequentemente e em determinados contextos sociodemográficos e, ao mesmo tempo, negligenciada e subestimada em outros 4,79. Essa situação foi descrita por Blum 26 como um paradoxo na prestação de cuidados nesse domínio em que fatores culturais, geográficos e socioeconômicos podem determinar uma desigualdade de acesso a serviços especializados de acordo com a sua localização e/ou disponibilidade nos setores público e privado.

No caso português, o enquadramento da PHDA esteve dependente de um processo de ampliação do campo diagnóstico e de intervenção no qual se aliam as unidades de saúde e hospitais universitários aos centros de desenvolvimento e de pesquisa 3. Esse processo culminou na criação, em 2017, da Sociedade Portuguesa de Défice de Atenção (SPDA, ver detalhes em https://spda.pt) que congrega profissionais de saúde e pesquisadores neste domínio. Em particular, a neuropsicologia tem desempenhado um papel importante na construção e validação de questionários, escalas psicométricas e protocolos de avaliação cognitiva-comportamental, bem como na sua adaptação e aferição para a população portuguesa 80,81,82. No estudo etnográfico antes referido, a avaliação e o diagnóstico da PHDA eram feitos por pediatras e psicólogos, por meio de um protocolo de avaliação específico que incluía, a título de exemplo, a entrevista simplificada com base nos critérios do DSM e os questionários de Conners, além das escalas de autoconceito e de inteligência de Wechsler para crianças 7 . A combinação desses instrumentos na prática clínica permite aos especialistas uma compreensão mais aprofundada e transversal dos problemas que estão frequentemente associados à PHDA, incluindo problemas relacionais e de baixa autoestima, transtornos de sono e bullying no contexto escolar 83,84,85, indo além dos critérios diagnósticos estipulados pelo DSM 7. Essa compreensão requer, necessariamente, uma abordagem dimensional e multifatorial aos problemas de desenvolvimento e de saúde mental que integre as suas dimensões relacionais e sociais e que assente em intervenções multimodais ao longo da vida 67,86 . 


\section{Discussão: desafios e implicações da PHDA para a saúde mental e global}

Com base em uma análise aprofundada e interdisciplinar, este artigo traçou o retrato do diagnóstico e tratamento da PHDA em Portugal nas suas vertentes históricas, socioéticas e institucionais. Nesse retrato, caracterizado por diversos elementos de complexidade, particularidade e até mesmo paradoxo, destacam-se três elementos. Primeiro, a noção de que a hiperatividade e déficit de atenção constituem um problema clinicamente complexo e relevante para a saúde pública global, mas que tem sido escassamente estudado no âmbito das ciências sociais e humanas. Em segundo lugar, as dinâmicas de ativismo e de contestação que caracterizam a legitimação e popularização do seu diagnóstico em Portugal, apesar da sua recente reconfiguração enquanto objeto de debate público e político. Em terceiro, a introdução precoce dos psicoestimulantes como tratamento de primeira linha da PHDA e a sua evolução recente no mercado português, paralelamente à ampliação dos meios diagnósticos e abordagens terapêuticas na interseção da pesquisa e prática clínicas e dos setores público e privado 3 .

O estudo de caso português permite-nos, dessa perspectiva, compreender a trajetória social da definição, contestação e ampliação da PHDA vista como processo dinâmico e situado, no qual se sobrepõem os sistemas internacionais de categorização diagnóstica e os dispositivos farmacêuticos aos enquadramentos institucionais, políticas públicas e práticas locais. Se esse processo parece refletir dinâmicas globais de medicalização e de expansão do diagnóstico e tratamento da PHDA, tal como vem sendo descrito na literatura da sociologia médica 45,87 , ele evidencia o papel desempenhado por profissionais de saúde e de educação e pela mídia local na abertura do debate público sobre esses temas. Além disso, salienta-se o papel de fatores sociais e estruturais que determinam a prestação de apoios às famílias e o seu acesso a diferentes modelos de cuidado e serviços especializados, os quais podem ajudar a explicar o crescimento e a predominância das intervenções farmacológicas com psicoestimulantes (p.ex.: o metilfenidato). Entre esses fatores denota-se a forma como a inserção socioeconômica e geográfica de Portugal no espaço europeu influenciou a disponibilização e evolução do consumo de psicoestimulantes no país, além da sua convergência com a implementação de medidas de austeridade financeira e os seus efeitos perniciosos na prestação de cuidados e na saúde mental dos portugueses ao longo da última década $57,88,89$.

Nos próximos anos, um dos desafios colocados pela PHDA em Portugal e no panorama global será o de como responder a problemas crônicos e necessidades especiais e/ou complexas na interseção do desenvolvimento infantil e da saúde mental, os quais se redefinem como condições para a vida toda. Essa redefinição requer, por sua vez, o desenvolvimento de novas orientações na prestação de cuidados continuados e integrados que englobem o meio clínico, familiar, social e comunitário, desde programas de intervenção precoce às estratégias de prevenção e promoção da saúde mental. Segundo o relatório de 2019 do CNS 67, essas orientações constituem um dos maiores desafios desta década na reforma da saúde mental em Portugal e uma reflexão-chave na implementação das agendas em saúde mental global, como sugerido recentemente por Caldas de Almeida 86. Para responder a esses desafios, devem desenvolver-se novas linhas de pesquisa social e interdisciplinar sobre a PHDA, além de estudos comparativos e longitudinais que iluminem padrões e variações demográficas, socioeconômicas e geográficas da sua prevalência, distribuição e tratamento. Afigura-se também a necessidade premente de um debate público informado que reúna profissionais, pesquisadores e decisores, assim como cientistas e trabalhadores sociais, professores e técnicos de educação e terapeutas. Esse debate deverá ser ampliado no sentido de reconhecer as necessidades dos cuidadores, pais e famílias e de incluir as vozes e experiências vividas pelas crianças, adolescentes e jovens adultos, como vem sendo defendido na literatura sobre a participação dos jovens e a ética do cuidado em saúde 4,90.

Com base no estudo aprofundado do caso português, o presente artigo avança com uma perspectiva situada e abrangente sobre a PHDA, que procura contextualizar tanto a especificidade da sua trajetória histórica e social quanto a transversalidade deste tema aos campos da saúde mental, pública e global. Por um lado, essa perspectiva permite-nos compreender a complexidade da PHDA na medida em que ela extravasa fronteiras nacionais e categorias diagnósticas cruzando determinantes sociais da saúde e a governação de políticas públicas em setores tão diversos quanto os da educação e ensino, da saúde mental e do medicamento e dos apoios sociais às crianças, jovens e famílias. Por outro, essa perspectiva ressalta a importância da análise histórica, sociológica e antropológica do diagnóstico, tratamento e epidemiologia da PHDA, para além da tese clássica da medicalização. Este 
artigo procura evidenciar a importância conceitual e a significância empírica das ciências sociais e humanas no desenvolvimento e reforma de programas para a saúde mental e global. Esses programas deverão assentar numa visão ética e multidisciplinar sobre as determinantes da saúde mental, como referido por Bemme \& Kirmayer 91, e num modelo conceitual não dicotômico da saúde pública global (p.ex.: o foro médico versus o social, o Norte e o Sul Global, o perito e o leigo) como sugerem Vincanne Adams et al. 92. Situar o déficit de atenção e hiperatividade implica uma perspectiva interdisciplinar e coletiva, que nos permita repensar tanto o contexto como o escopo sociais da saúde mental e da saúde pública global.

\section{Informação adicional}

ORCID: Angela Marques Filipe (0000-0002-78997336).

\section{Agradecimentos}

Uma palavra de agradecimento a todos os participantes, colaboradores e colegas que tornaram este trabalho possível. Obrigada também aos revisores pelos seus comentários construtivos e à Alexandra Silva, Sofia Silva, Olga Marques e Cátia Duarte pelas suas sugestões na revisão de texto. Este artigo representa, exclusivamente, as perspectivas e a pesquisa do/a autor/a que não reporta quaisquer conflitos de interesse. Os autores/as e organizações mencionadas neste artigo não são responsáveis, de forma alguma, pelos argumentos e/ou conteúdos nele apresentados.

\section{Referências}

1. Polanczyk GV, Willcutt EG, Salum GA, Kieling $\mathrm{C}$, Rohde LA. ADHD prevalence estimates across three decades: an updated systematic review and meta-regression analysis. Int J Epidemiol 2014; 43:434-42.

2. Thomas R, Sanders S, Doust J, Beller E, Glasziou P. Prevalence of attention-deficit/hyperactivity disorder: a systematic review and metaanalysis. Pediatrics 2015; 135:994-1001.

3. Filipe AM. The emergence and shaping of ADHD in Portugal: ambiguities of a diagnosis "in the making". In: Bergey M, Filipe AM, Conrad P, Singh I, editores. Global perspectives on ADHD: social dimensions of diagnosis and treatment in sixteen countries. Baltimore: Johns Hopkins University Press; 2018. p. 11837.

4. Singh I, Filipe AM, Bard I, Bergey M, Baker L. Globalization and cognitive enhancement: emerging social and ethical challenges for ADHD clinicians. Curr Psychiatry Rep 2013; 15:385.

5. Carreira A. Hiperatividade é a perturbação mais diagnosticada nas crianças em Portugal. Diário de Notícias 2010; 18 mai. https://www. dn.pt/portugal/hiperactividade-e-a-perturba cao-mais-diagnosticada-1572761.html.

6. Boavida J, Estrada M. Perturbação de hiperatividade e défice de atenção: quando a ignorância faz vítimas inocentes. Observador 2019; 29 jul. https://observador.pt/opiniao/pertur bacao-de-hiperatividade-e-defice-de-atencaoquando-a-ignorancia-faz-vitimas-inocentes/.

7. Filipe AM. Making ADHD evident: data, practices, and diagnostic protocols in Portugal. Med Anthropol 2016; 35:390-403. 
8. Appadurai A, editor. The social life of things: commodities in cultural perspective. Cambridge: Cambridge University Press; 1988.

9. Lima S. A psicologia em Portugal. Biblos 1949; 25:277-85.

10. Barahona Fernandes HB. A psiquiatria em Portugal. Lisboa: Roche Farmacêutica; 1984.

11. Mendonça MM. Mais vale prevenir... Memórias de uma época e de um contributo para a saúde mental infantil. Coimbra: Minerva; 2002.

12. Filipe AM. The rise of child psychiatry in Portugal: an intimate social and political history, 1915-1959. Soc Hist Med 2014; 27:326-48.

13. Sardica J. Twentieth Century Portugal. A historical overview. Lisboa: Universidade Católica Editora; 2008.

14. Pinto Costa R. O poder médico no Estado Novo (1945-1974). Porto: Universidade do Porto Editora; 2009.

15. Hespanha P. A reforma psiquiátrica em Portugal: desafios e impasses. In: Fontes B, Fonte E, editores. Desinstitucionalização, redes sociais e saúde mental: análise de experiências da reforma psiquiátrica em Angola, Brasil e Portugal. Recife: Editora Universitária da UFPE; 2010. p. 137-61.

16. Ministério da Saúde. Rede de referenciação hospitalar de psiquiatria da infância e da adolescência. http://www.acss.min-saude.pt/ Portals/0/DOCUMENTO\%20T\%C3\%89C NICO\%20DE\%20SUPORTE_RRH_PSIQ\%20 IA_VERS\%C3\%83O_\%2023\%20NOV.pdf (acessado em 26/Nov/2012).

17. Barahona Fernandes H. Classificações internacionais e diagnóstico individual estruturado e personalizado. A propósito de um caso de "fronteira". O Médico 1982; 1591:857-75.

18. Barahona Fernandes H, Polónio P, Seabra-Dinis J. Classificação internacional das doenças mentais. Proposta de adaptação portuguesa. Jornal do Médico 1970; 1428:300-8.

19. Ataíde JS. Elementos de psiquiatria da criança e do adolescente. Lisboa: Instituto de Assistência Psiquiátrica e Centro de Saúde Mental Infantil de Lisboa; 1977.

20. Lakoff A. Adaptive will: the evolution of attention deficit disorder. J Hist Behav Sci 2000; 36:149-69.

21. Rebelo J. Para uma delimitação da noção de criança hiperactiva. Revista Portuguesa de Pedagogia 1986; 20:203-18.

22. Serrão Neto A. Hiperatividade e défice de atenção. Lisboa: Verso de Kapa; 2014.

23. Oliveira G, Duque F, Duarte C, Melo F, Teles L, Brito M, et al. Pediatria do neurodesenvolvimento. Levantamento nacional de recursos e necessidades. Acta Pediátrica Portuguesa 2012; 43:1-17.

24. American Psychiatric Association. Diagnostic and statistical manual of mental disorders. 5th Ed. Washington DC: American Psychiatric Publishing; 2013.
25. Edwards C, Howlett E, Akrich M, Rabeharisoa V. Attention deficit hyperactivity disorder in France and Ireland: parents' groups' scientific and political framing of an unsettled condition. BioSocieties 2014; 9:153-72.

26. Blum LM. Raising generation Rx: mothering kids with invisible disabilities in an age of inequality. New York: New York University Press; 2015.

27. Singh I. Bad boys, good mothers, and the "miracle" of ritalin. Sci Context 2002; 15:577-603.

28. Singh I. Boys will be boys: fathers' perspectives on ADHD symptoms, diagnosis, and drug treatment. Harv Rev Psychiatry 2003; 11:30816.

29. Bowker G, Berg M. The multiple bodies of the medical record: towards a sociology of an artifact. Sociol Q 1997; 38:513-37.

30. Mol A. The body multiple: ontology in medical practice. Durham: Duke University Press; 2002.

31. Ferreira JC. Perspectiva histórica da PHDA. In: Serrão Neto A, editor. Hiperatividade e défice de atenção. Lisboa: Verso de Kapa; 2014. p. 1521.

32. Fonseca A, Simões M, Rebelo J, Borges L, Boavida Fernandes J, Oliveira G, et al. Hiperactividade na comunidade e hiperactividade em meio clínico: semelhanças e diferenças. Psychologica 1998; 19:111-22.

33. Barkley RA. Behavioral inhibition, sustained attention, and executive functions: constructing a unifying theory of ADHD. Psychol Bull 1997; 121:65-94.

34. Barkley RA. International consensus statement on ADHD. Clin Child Fam Psychol Rev 2002; 5:89-111.

35. Infarmed: Autoridade Nacional do Medicamento e Produtos de Saúde. Hiperatividade: análise à utilização de medicamentos em Portugal continental. http://www.infarmed. $\mathrm{pt} / \mathrm{portal} / \mathrm{page} / \mathrm{portal} / \mathrm{INFARMED/PUBLI}$ CACOES/INFARMED_NOTICIAS/infar med\%20not\%EDcias\%20N.\%BA\%2054\%20 -\%20maio\%202015_1.pdf (acessado em 27/ Mai/2015).

36. Ministério da Justiça. Decreto-Lei no 15 de 22 Janeiro de 1993. https://data.dre.pt/eli/declei/15/1993/01/22/p/dre/pt/html.

37. Porfírio H, Boavida Fernandes J, Borges L. Intervenção psicofarmacológica na perturbação por défice de atenção com hiperactividade. Psychologica 1998; 19:201-7.

38. Rose N. Disorders without borders? The expanding scope of psychiatric practice. BioSocieties 2006; 1:465-84.

39. Singh I. Beyond polemics: science and ethics of ADHD. Nat Rev Neurosci 2008; 9:957-64.

40. Parens E, Johnston J. Facts, values, and attention-deficit hyperactivity disorder (ADHD): an update on the controversies. Child and Adolescent Psychiatry and Mental Health 2009; 3:1. 
41. Mota HC. Bichos carpinteiros normalizados. Revista Saúde Infantil 1999; 21:69-70.

42. Boavida J. Hiperactividade ou má 'educação'? Saúde Infantil 2006; 28:3-5.

43. Rosenberg C. The tyranny of diagnosis: specific entities and individual experience. Milbank Q 2002; 80:237-60.

44. Conrad P. Identifying hyperactive children: the medicalization of deviant behavior. Aldershot/Burlington: Ashgate Publishing; 2006.

45. Lusardi R. Current trends in medicalisation: universalising ADHD diagnosis and treatments. Sociol Compass 2019; 13:e12697.

46. Cordinhã AC, Boavida J. A criança hiperactiva: diagnóstico, avaliação e intervenção. Revista Portuguesa de Medicina Geral 2008; 24:57789.

47. Padilhão C, Marques M, Marques C. Perturbações do comportamento e perturbação de hiperactividade com défice de atenção: diagnóstico e intervenção nos cuidados de saúde primários. Revista Portuguesa de Clínica Geral 2009; 25:592-9.

48. Rodrigues A, Nuno Antunes N. Mais forte do que eu! Hiperactividade e défice de atenção: causas, consequências e soluções. Lisboa: Lua de Papel; 2014.

49. Rafalovich A. Attention deficit-hyperactivity disorder as the medicalization of childhood: challenges from and for sociology. Sociol Compass 2013; 7:343-54.

50. Malacrida C. Medicalization, ambivalence and social control: mothers' descriptions of educators and ADD/ADHD. Health (NY) 2004; 8:6180.

51. Rojas Navarro S, Vrecko S. Pharmaceutical entanglements: an analysis of the multiple determinants of ADHD medication effects in a Chilean school. Int J Qual Stud Health Wellbeing 2017; 12 Suppl 1:1298268.

52. Ministério da Educação. Decreto-Lei no 3, de 7 de janeiro de 2008. http://legislacao.min-edu.pt/np4/np3content/?newsId=1530\&fileNa me=decreto_lei_3_2008.pdf (acessado em 25/ $\mathrm{Jul} / 2013$ ).

53. Provedor da Justiça. Subsídio por frequência de estabelecimento de educação especial. Recomendação no 1/A/2008 2008. http://www. provedor-jus.pt $/$ ?action $=5 \&$ idc $=67 \&$ idi $=1116$ (acessado em 23/Jul/2013).

54. Pais SC, Menezes I, Nunes JA. Saúde e escola: reflexões em torno da medicalização da educação. Cad Saúde Pública 2016; 32:e00166215.

55. Infarmed: Autoridade Nacional do Medicamento e Produtos de Saúde. Informação aos profissionais de saúde: recomendações relativas à segurança do Metilfenidato. http://www. infarmed.pt/infarmedia/47/infarmedia.pdf (acessado em 02/Set/2014).

56. Diário da República. Aviso no 3775/2009. https://dre.pt/application/dir/pdf2s/2009/ 02/033000000/0662506628.pdf (acessado em 02/Set/2014).
57. Barradas C, Nunes JA. Facing austerity: the decline in health access and quality of care for patients with cancer in Portugal. Hist Ciênc Saúde Manguinhos 2017; 24:933-51.

58. Observatório Português dos Sistemas de Saúde. Relatório de primavera 2013. Duas faces da saúde. Coimbra: Observatório Português dos Sistemas de Saúde; 2013.

59. Palha M. Pediatra denuncia ruptura de stock de medicamento para a concentração. Público 2012; 3 mai. https://www.publico. pt/2012/05/03/sociedade/noticia/pediatradenuncia-ruptura-de-stock-de-medicamento -para-a-concentracao-1544535.

60. Vendas de medicamentos para concentração aumentaram 78\% em cinco anos. Jornal i 2012; 4 mai. http://www.ionline.pt/artigos/portu gal/vendas-medicamentos-concentracao-au mentaram-78-cinco-anos.

61. Fabião C, Menezes I, Coimbra J, Carvalho MI, Silva M, Sousa ML, et al. Manifesto: por uma abordagem não medicalizante nem patologizante da educação. http://educationme dicalisation.blogspot.com (acessado em 18/ Mar/2013).

62. Borges L. Faltam estruturas para tratar hiperactividade e défice de atenção em Portugal. Notícias da Saúde em Portugal 2006; 15 nov. http://cybersaude.wordpress. com/2006/11/15/faltam-estruturas-para-tra tar-hiperactividade-e-defice-de-atencao-emportugal/.

63. Gomes Pedro J. Hiperatividade está mal diagnosticada em Portugal. Diário de Notícias 2015; 23 mar. http://www.dn.pt/inicio/por tugal/interior.aspx?content_id=4469884\&pa ge $=2$.

64. Programa Nacional para a Saúde Mental, Direção-Geral da Saúde. Relatório do Programa Nacional para a Saúde Mental 2017. https:// www.dgs.pt/em-destaque/relatorio-do-pro grama-nacional-para-a-saude-mental-2017. aspx (acessado em 17/Mar/2019).

65. European Medicines Agency. Assessment report pursuant to Article 29(4) of Directive 2001/83/EC. https://www.ema.europa.eu/en/ documents/referral/methylphenidate-sandozarticle-29-referral-assessment-report_en.pdf (acessado em 02/Set/2014).

66. Infarmed: Autoridade Nacional do Medicamento e Produtos de Saúde; Sistema Nacional de Saúde. Relatório de avaliação do pedido de comparticipação de medicamento para uso humano - Dimesilato de Lisdexanfetamina. https://www. infarmed.pt/documents/15786/1437513/ Relatório+público+de+avaliação $+\mathrm{de}+\mathrm{El}$ vanse $+\% 28$ dimesilato + de + lisdexanfeta mina\%29+2019/2a2e2d48-70d3-4177-9c0c966ed00307ed (acessado em 05/Jan/2020). 
67. Conselho Nacional de Saúde. Sem mais tempo a perder: saúde mental em Portugal - um desafio para a próxima década. Lisboa: Ministério da Saúde/Conselho Nacional de Saúde; 2019.

68. Soeiro J. Toma o comprimido e cala-te? Expresso 2015; 29 mai. http://expresso.sapo.pt/ blogues/jose-soeiro/2015-05-29-Toma-ocomprimido-e-cala-te.

69. Bento H. PAN quer que medicamentos para a hiperatividade e défice de atenção nas crianças sejam receitados apenas por especialistas. Expresso 2019; 22 abr. https://expresso.pt/ sociedade/2019-04-22-PAN-quer-que-medi camentos-para-a-hiperatividade-e-defice-de -atencao-nas-criancas-sejam-receitados-ape nas-por-especialistas.

70. Campos A. Vendas de fármacos para hiperactividade baixam. É um bom sinal? Público 2018; 27 jan. https://www.publico.pt/2018/01/27/ sociedade/noticia/vendas-de-farmacos-para-hiperactividade-baixam-isso-e-um-bom-si nal-1800933.

71. Coordenação Nacional para a Saúde Mental. Recomendações para a prática clínica da saúde mental infantil e juvenil nos cuidados de saúde primários. Lisboa: Ministério da Saúde; 2009.

72. Caldas de Almeida JM, Xavier M, Cardoso G, Pereira MG, Gusmão R, Corrêa B, et al. Estudo epidemiológico nacional de saúde mental. http://www.fcm.unl.pt/main/alldoc/galeria_ imagens/Relatorio_Estudo_Saude-Mental_2. pdf. (acessado em 18/Mai/2015).

73. National Institute for Health and Care Excellence. Attention deficit hyperactivity disorder: diagnosis and management. https://www. nice.org.uk/guidance/ng87 (acessado em 17/ Nov/2018).

74. Wolraich ML, Hagan JF, Allan C, Chan E, Davison $\mathrm{D}$, Earls $\mathrm{M}$, et al. Clinical practice guideline for the diagnosis, evaluation, and treatment of attention-deficit/hyperactivity disorder in children and adolescents. Pediatrics 2019; 144:e20192528.

75. Marques MI, Matias J, Machado R, Duarte A, Fonseca MJ, Monteiro JP. Perturbação de hiperatividade e défice de atenção em idade pediátrica: papel dos cuidados de saúde primários. Gaz Médica 2018; 5:28-36.

76. Ordem e DGS preparam normas para tratamento de crianças com défice de atenção. Diário Notícias 2018, 2 out. https://www.dn.pt/ vida-e-futuro/ordem-e-dgs-preparam-nor mas-para-tratamento-de-criancas-com-defi ce-de-atencao-9946537.html.

77. Ordem dos Médicos. Parecer sobre o tratamento da perturbação de hiperatividade e défice de atenção. http://ordemdosmedicos. pt/wp-content/uploads/2017/09/PARECER MEDICAMENTOS_PARA_PHDA.pdf (acessado em 18/Mar/2019).

78. Filipe C. A perturbação de hiperactividade com défice de atenção no adulto. Revista Portuguesa de Clínica Geral 2004; 20:733-7.
79. Parens E. On good and bad forms of medicalization. Bioethics 2013; 27:28-35.

80. Rodrigues AN. Escalas revistas de Conners: formas reduzidas para pais e professores. In: Simões M, Machado C, Gonçalves M, Almeida L, organizadores. Aval psicológica: instrumentos validados para a população portuguesa. Coimbra: Quarteto Editora; 2007. p. 203-26.

81. Alfaiate C. Impacto da perturbação de hiperactividade com défice de atenção (subtipo Combinado) no funcionamento neuropsicológico: estudos de validade com a bateria de avaliação neuropsicológica de Coimbra [Dissertação de Mestrado]. Coimbra: Faculdade de Psicologia e de Ciências da Educação, Universidade de Coimbra; 2009.

82. Parreira AF, Martins A, Ribeiro F, Silva FG. Clinical validation of the Portuguese version of the Children's Sleep Habits Questionnaire (CSHQ-PT) in children with sleep disorder and ADHD. Acta Med Port 2019; 32:195-201.

83. Maia C, Guardiano M, Viana V, Almeida JP, Guimarães MJ. Auto-conceito em crianças com hiperactividade e défice de atenção. Acta Med Port 2011; 24:493-502.

84. Oliveira L, Pereira M, Serrano A, Medeiros T. PHDA em contexto escolar: comorbilidades e problemas de desempenho associados. Revista E-Psi 2017; 7:77-100.

85. Cabral P. Attention deficit disorders: are we barking up the wrong tree? Eur J Paediatr Neurol 2006; 10:66-77.

86. Caldas de Almeida JM. Four reflections on the new global mental health priorities. Epidemiol Psychiatr Sci 2020; 29:e75.

87. Conrad P, Bergey MR. The impending globalization of ADHD: notes on the expansion and growth of a medicalized disorder. Soc Sci Med 2014; 122:31-43.

88. Augusto GF. Mental health in Portugal in times of austerity. Lancet Psychiatry 2014; 1:109-10.

89. Legido-Quigley H, Karanikolos M, Hernandez-Plaza S, Freitas C, Bernardo L, Padilla B, et al. Effects of the financial crisis and Troika austerity measures on health and health care access in Portugal. Health Policy 2016; 120:833-9.

90. Ferreira P, Filipe AM. Bioética. https://alice. ces.uc.pt $/$ dictionary $/$ ?id $=23838 \&$ pag $=23918 \&$ id_lingua $=2 \&$ entry $=24616$ (acessado em 01/ $\mathrm{Abr} / 2019)$

91. Bemme D, Kirmayer LJ. Global Mental Health: interdisciplinary challenges for a field in motion. Transcult Psychiatry 2020; 57:3-18.

92. Adams V, Behague D, Caduff C, Löwy I, Ortega F. Re-imagining global health through social medicine. Glob Public Health 2019; 14:1383400. 


\section{Abstract}

Attention deficit and hyperactivity disorder $(A D H D)$ is considered one of the most frequent behavioral and neurodevelopmental problems in school-age children and adolescents, both in Portugal and worldwide. The diagnostic categorization of ADHD and the prescription of psychostimulants as its first-line treatment have been the object not only of scientific research and clinical validation, but also of controversy and social critique, especially in light of the concept of medicalization. Despite its high profile and salience in such diverse fields as education, pharmaceuticals, mental health, and public policy, a significant gap remains in the characterization of social-historical, ethical, and institutional dimensions of $A D H D$ outside English-speaking countries. Combining historical and ethnographic research with document and media analysis, the article addresses that challenge by tracing the social trajectory of $A D H D$ in Portugal, from the emergence of "hiperactivity" in the 1970s and 1980s to the current public and political debates on psychostimulant treatments and prescribing trends. From this interdisciplinary perspective and based on the Portuguese case study, the aim of this article is to contextualize the definition, validation, and expansion of $A D H D$ as part of a dynamic and socially situated process in which global diagnostic and pharmaceutical systems intersect with institutional and socioeconomic contingencies, as well as local specificities and needs. More broadly, the article discusses how the case study of ADHD contributes to the development of interdisciplinary research that helps rethinking the social scope of mental health across local and global health contexts.

Attention Deficit Disorder with Hyperactivity; Public Policy; Global Health; Mental Health; Social Sciences

\section{Resumen}

El trastorno de hiperactividad y déficit de atención (THDA) está considerado uno de los problemas de comportamiento y neurodesarrollo más frecuentes en niños $y$ adolescentes en edad escolar, tanto en Portugal, como a nivel mundial. Asimismo, la categorización diagnóstica del THDA y la prescripción de psicoestimulantes como tratamiento de primera línea ha sido no sólo objeto de investigación científica y validación clínica, sino también objeto de polémica y crítica social, sobre todo a la luz del concepto de medicalización. A pesar de su cariz mediático y relevancia en dominios tan diversos como son los de la educación, del medicamento, de la salud mental y de los apoyos psicosociales, existe una laguna profunda en la caracterización de las dimensiones históricas, socio-éticas e institucionales del THDA en Portugal. Aunando datos historiográficos y etnográficos y al análisis documental, y de los medios de comunicación, este artículo procura responder a este desafío, trazando la trayectoria social del THDA en este país desde la emergencia de la "hiperactividad" en los años 1970/1980 al debate público y político sobre la prescripción de psicoestimulantes. Desde esta perspectiva interdisciplinar, $y$ a través del estudio del caso portugués, se intenta contextualizar la definición, validación y ampliación del THDA como parte de un proceso dinámico y socialmente situado, que atraviesa sistemas diagnósticos y farmacéuticos globales, contingencias institucionales $y$ socioeconómicas e, incluso, politicas públicas y especificidades locales. Se discute, finalmente, la forma en la que el caso del THDA en Portugal contribuye al desarrollo de nuevas líneas de reflexión $y$ de investigación interdisciplinaria que permiten repensar el campo social de la salud mental y de la salud global.

Trastorno por Déficit de Atención con Hiperactividad; Política Pública; Salud Global; Salud Mental; Ciencias Sociales
Recebido em 23/Mar/2020

Versão final reapresentada em 30/Ago/2020 Aprovado em 01/Out/2020 